\title{
Measuring Surface Tension of III-V Nanowire Au-Catalyst Droplets with an E-field
}

\author{
$\underline{\text { Christopher Røhl Andersen }}^{1,2,3}$, Marcus Tornberg ${ }^{2,3,4}$, Daniel Jacobsson ${ }^{2,3}$, Kimberly A. Dick ${ }^{2,3,4}$ and \\ Kristian Mølhave ${ }^{1}$
}

${ }^{1}$ National Centre for Nano Fabrication and Characterization, Technical University of Denmark, 2800

Kgs. Lyngby, Denmark.

${ }^{2}$ nCHREM and Centre for Analysis and Synthesis, Lund University, 22100 Lund, Sweden.

${ }^{3}$ NanoLund, Lund University, 22100 Lund, Sweden.

${ }^{4}$ Solid State Physics, Lund University, 22100 Lund, Sweden.

Liquid droplets hold together by the surface tension, which is the force that makes the surface molecules attract each other. It is not only the phenomenon explaining droplet formation, but it is also an important factor, when studying vapor solid liquid (VLS) growth, which is a common used growth mechanism for nanowires [1].

Controlling crystal growth including the formation of nanostructures are of great importance for developing modern technology such as optoelectronics and quantum transport [2]. Today however, growth models and predictions are based on approximate values of the surface energy lacking in reports of empirical values [3].

Previous studies by F. Panciera et al have used special designed silicon cantilever chips to determine the surface tension of AuSi eutectic droplets on a silicon nanowire by applying a bias between two opposing cantilevers [4]. In our study, we use the same procedure on Au-catalyzed III-V nanowires grown on silicon cantilevers deforming Au-III eutectic droplets in an arsine atmosphere. The study is performed in a unique ETEM with a purpose built gas handling system for metal-organic vapor deposition [5].

Au-catalyzed GaAs nanowires are nucleated and grown epitaxially from the silicon (111) cantilever acting as the substrate as illustrated in figure 1. Growth is terminated by stopping flow of Ga precursor gas as the nanowires are close to the opposing cantilever with a distance of up to $800 \mathrm{~nm}$, where the eutectic droplet can be deformed by applying an increasing bias between the two cantilevers as shown in figure 2 . The change in the droplet curvature is compared with numerical models and the surface tension is found from the best fit of the empirical and numerical results.

The numerical models are based on two iterative steps calculating the electrical potential by solving the Laplace equation and using the electrical potential for solving the Young-Laplace equation to calculate the normal stress balance until the droplet shape has stabilized [6].

In conclusion, we present empirical values of the surface tension of the Au-catalyzed III-V nanowire eutectic droplets using E-field deformation in an environmental TEM. 


\section{References:}

[1] RS Wagner and WC Ellis, Appl. Phys. Lett. 4 (1964), p. 89.

[2] X Dai et al in "Semiconductor Nanowires: Materials, Synthesis, Characterization and Applications", ed. J. Arbiol and Q. Xiong, (Woodhead Publishing, 2015), p. 71.

[3] EK Mårtensson et al, Nano Lett. (2019), p. 1197.

[4] F Panciera et al, Nat. Commun. 7 (2016), p. 12271.

[5] C Hetherington et al, Semicond Sci Technol 35 (2020), p. 34004.

[6] LT Corson et al, Phys Fluids 26 (2014), p. 122106.

[7] The authors acknowledge funding from DTU Nanolab, DTU Fotonik, the Knut and Alice

Wallenberg Foundation (KAW), the Swedish Research Council (VR), and the Swedish Foundation for Strategic Research (SSF).

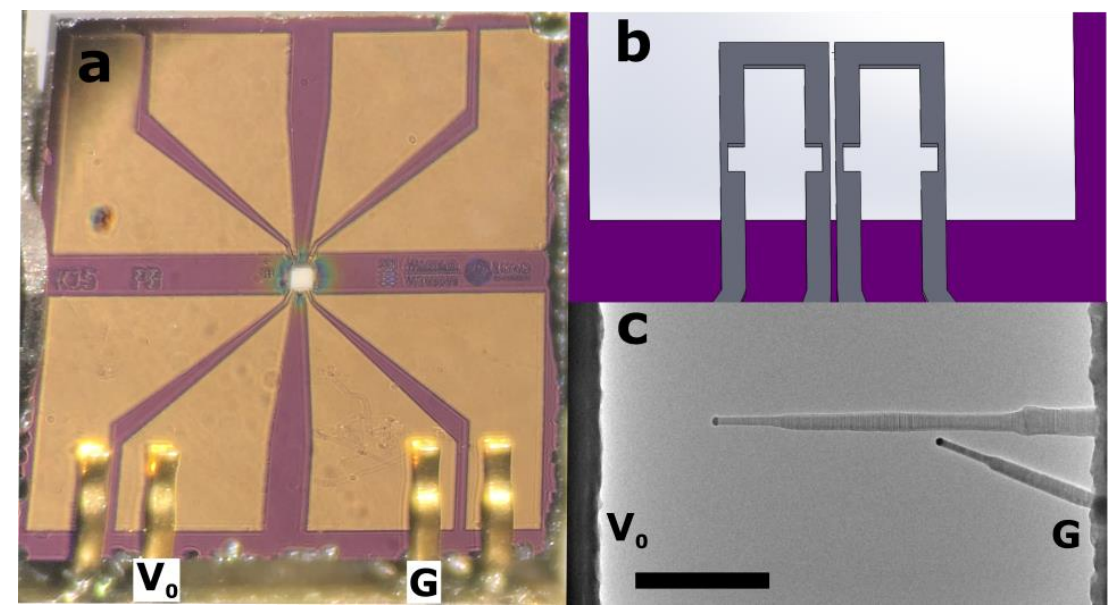

Figure 1. Overview of a chip loaded into a sample holder (a) and an illustration of the window in the center of the chip with a pair of cantilevers hanging into vacuum (b). The cantilevers are heated by resistive heating and Au-catalyzed III-V nanowires nucleate in-situ and grow epitaxially from one cantilever (c). A bias is applied between the two cantilevers to deform the droplet. Scale bar is $500 \mathrm{~nm}$.

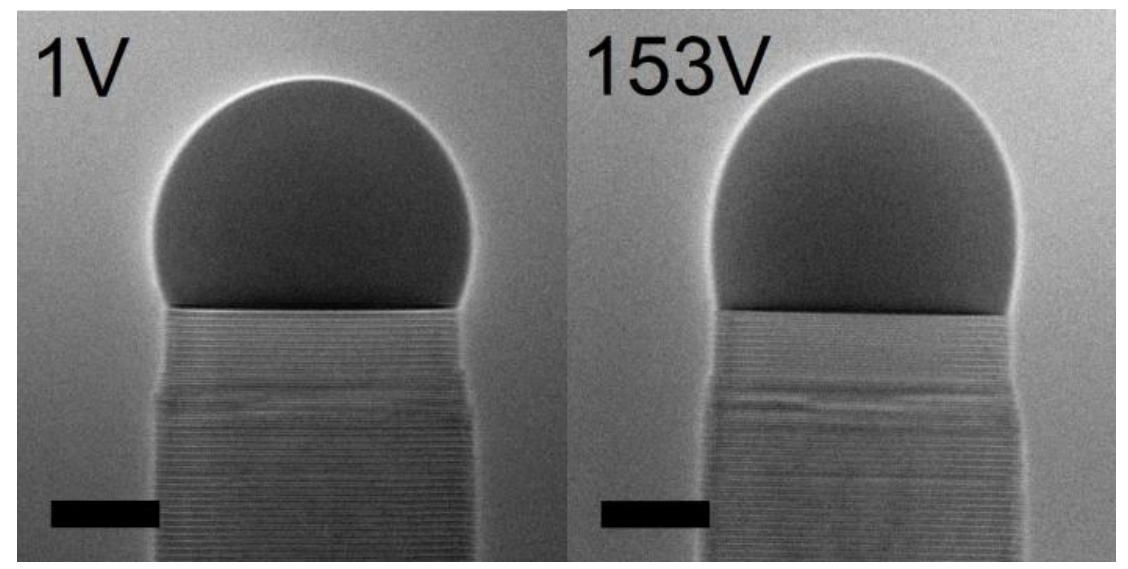

Figure 2. AuGa(SiIn) droplets are deformed by applying an electric bias, while the nanowire is annealed in an arsine atmosphere to avoid etching of the nanowire by the eutectic droplet. Scale bar is 5 . 